\title{
Susceptibility of invasive mosquitoes (Diptera: Culicidae) for infection with Japanese encephalitis virus, an emerging zoonosis
}

Luis Miguel Hernandez-Triana ( $\sim$ luis.hernandez-triana@apha.gov.uk)

Animal and Plant Health Agency

Luis Miguel Hernandez-Triana

Animal and Plant Health Agency https://orcid.org/0000-0001-7058-8848

Arran J. Folly

Animal and Plant Health Agency

Sanam Sewgobind

Animal and Plant Health Agency

Fabian Z. X. Lean

Animal and Plant Health Agency

Stuart Ackroyd

Animal and Plant Health Agency

Alejandro Nuñez

Animal and Plant Health Agency

Sarah Delancour

Universidad de Zaragoza Facultad de Veterinaria

Andrea Drago

Entostudio S. r. L.

Patrizia Visetin

Entostudio S.R.L.

Karen L. Mansfield

Animal and Plant Health Agency

Nicholas Johnson

Animal and Plant Health Agency

\section{Research Article}

Keywords: Mosquito, invasive species, Japanese encephalitis, zoonosis, vector competency, emerging infectious disease

Posted Date: February 24th, 2022 
DOI: https://doi.org/10.21203/rs.3.rs-1370565/v1

License: (c) (1) This work is licensed under a Creative Commons Attribution 4.0 International License. Read Full License 


\section{Abstract}

Background: Japanese encephalitis virus (JEV) is the principal cause of mosquito-borne encephalitis in human populations within Asia. If introduced into new geographic areas, it could have implications for public and animal health. However, potential mosquito vectors for virus transmission have not been fully investigated. The Asian tiger mosquito, Aedes albopictus, has emerged into Europe and is now expanding its geographical range into more northerly latitudes. Culex quinquefasciatus, although absent from Europe, has been detected in Turkey, a country with territory in Europe, and could act as a vector for JEV in other regions. To assess the risk of these invasive species acting as vectors for JEV, we have investigated the vector competence of Ae. albopictus and $C x$. quinquefasciatus

Methods: Two colonised lines of Ae. albopictus (Italy and Spain), and a line of Cx. quinquefasciatus (Tanzania) were compared for susceptibility to infection by oral feeding with JEV strain SA-14, genotype $\mathrm{III}$, at $10^{6} \mathrm{PFU} / \mathrm{ml}$ and maintained at $25^{\circ} \mathrm{C}$. Specimens were processed at 7 and 14 days post-infection (dpi). Rates of infection, dissemination and transmission were assessed through detection of viral RNA by real-time polymerase chain reaction (RT-PCR) in mosquito body, legs and saliva, respectively, at each time point. Where possible, infection and dissemination were confirmed by immunohistochemical (IHC) detection of the JEV envelope protein.

Results: Aedes albopictus from Italy showed no susceptibility to infection with JEV strain SA-14. Conversely, Ae. albopictus colonised in Spain was susceptible and $100 \%$ of infected mosquitoes expressed viral RNA in saliva at $14 \mathrm{dpi}$. Culex quinquefasciatus was highly susceptible to infection as early as $7 \mathrm{dpi}$ and $50 \%$ of infected mosquitoes expressed viral RNA in saliva at $14 \mathrm{dpi}$. Infection and dissemination were confirmed in $C x$. quinquefasciatus by IHC detection of JEV envelope protein in both the mid-gut and salivary glands.

Conclusions: Aedes albopictus from two different locations in Europe range from being fully susceptible to JEV and capable of transmission, through to being totally resistant. Culex quinquefasciatus, also appears highly susceptible, therefore both species could potentially act as competent vectors for JEV and facilitate the emergence of JEV into new regions.

\section{Background}

Flaviviruses have a global distribution and many species can be transmitted by mosquitoes, ticks and sandflies [1, 2]. Japanese encephalitis virus (JEV) (Family Flaviviridae, Genus Flavivirus) is the main aetiological agent for human viral encephalitis in Far East and South East Asia. Five genotypes are currently recognised, all endemic in Asia [3,4].

Japanese encephalitis virus is maintained in an enzootic cycle between mosquito vectors and avian hosts, especially wading birds [5], although recent infection studies have demonstrated that domestic birds may act as JEV reservoirs [6]. Some mosquito species can act as bridge vectors transmitting the virus to livestock animals such as pigs, which are considered an amplifying host. Humans and other 
mammals such as horses are considered dead-end hosts, and recent studies have shown that pig-to-pig transmission can occur by the oronasal infectious route $[5,7]$. Due to a combination of climate change, movement of people and livestock, and the introduction of invasive vectors into new areas, geographic expansion of JEV could occur in the future $[8,9]$.

Culex tritaeniorhynchus is the main mosquito vector of JEV transmission in regions where JEV is endemic, but other species, particularly within the genus Culex, can act as vectors $[3,10]$. In Europe, $C x$. tritaeniorhynchus has only been reported in Greece [11], and JEV RNA has only been detected in a pool of $C x$. pipiens in northern Italy [12]. Recent experimental studies have demonstrated the competence of European populations of mosquitoes to transmit JEV, which include $C X$. pipiens from the UK and France, as well as Culiseta annulata and Aedes detritus from the UK [13-15]. Vector competence studies carried out by de Wispelaere et al. [16] demonstrated that a French population of the invasive species $A e$. albopictus was competent for JEV genotypes III and V, and Huber et al. [17] showed that Ae. japonicus japonicus from Germany is also a competent JEV vector. As a consequence, it is likely that there are many unassessed mosquito vectors with endemic European populations that may be able to transmit JEV and facilitate its emergence. In addition, non-native species to Europe have the potential to act as JEV vectors. These include $C x$. quinquefasciatus, which has been found in Turkey [18], and Ae. albopictus and Ae. japonicus japonicus, which are now widely distributed in countries around the Mediterranean basin $[19,20]$. The detection of $C x$. quinquefasciatus in Turkey could enable this mosquito species to expand its geographical range into continental Europe in the near future. In other regions, different populations of Ae. albopictus have been identified as competent vectors of JEV such as Australia and Taiwan [21-23], and Cx. quinquefasciatus in North America [24], Brazil [13], India [25] and a colony from Queensland in Australia [26] were also competent to transmit JEV. However, other studies have shown that a wild caught population of $C x$. quinquefasciatus from Australia and New Zealand were not competent to transmit JEV [26, 27], and two strains of Ae. albopictus (Yungho and Liyang, Taichung County) were less efficient vectors in comparison with a strain originating from Sanhsia (Taipei County) from Taiwan.

Due to the continued risk of invasive mosquito species globally, and the potential emergence of JEV from regions in which it is endemic, this study assessed the vector competence of two populations of $A e$. albopictus (originating from Italy and Spain), and $C x$. quinquefasciatus (originating from Tanzania) for JEV genotype III.

\section{Methods}

\section{Colonization of mosquitoes}

Laboratory colonies of Ae. albopictus (Padua, Italy) (year of colonization unknown and donated by Entostudio, Italy), Ae. albopictus (Barcelona, year of colonization 2009 and donated by Universidad de Zaragoza, Spain) (Figure 1), and Cx. quinquefasciatus (established at the Tropical Pesticides Research Institute (TPRI), Arusha, East Tanzania)(year of colonization at London School of Hygiene and Tropical 
Medicine 2010 and donated by London School of Hygiene and Tropical Medicine, UK). Maintenance of Culex and Aedes mosquitoes in biosecurity level 3 laboratories followed previously published protocols $[19,28]$.

\section{Virus stocks}

Japanese Encephalitis virus genotype III (strain SA-14, isolated from Cx. pipiens larvae, China 1954) was donated by Dr. Jonas Schmidt-Chanasit, Bernhard Nocht Institute for Tropical Medicine, Hamburg, Germany). Virus stocks were propagated in Vero cells as previously described $[15,19,28]$.

\section{Assessment of vector competence}

Adult females of Ae. albopictus (Italy and Spain) and Cx. quinquefasciatus (Tanzania) were tested for their vector competence for JEV genotype III at $25^{\circ} \mathrm{C}$. Mosquitoes were provided with $1.5 \mathrm{ml}$ of an infectious blood meal composed of defibrinated horse blood, adenosine 5 '-triphosphate (final concentration $0.02 \mathrm{mM}$ ) and virus stock to give a final virus concentration of $3.3 \times 10^{6} \mathrm{PFU} / \mathrm{ml}$, using a Hemotek membrane feeding system (Hemotek Ltd Accrington, Lancashire, UK). Five to ten-day-old adult female mosquitoes of both species were first starved of sucrose for five hours and then allowed to feed on the infectious bloodmeal (as described above) in Bugdorm insect cages of $22 \times 22 \times 22 \mathrm{~cm}$ (bugzarre.co.uk) from 16:00, continuing throughout the night for a minimum of $16 \mathrm{~h}$. The following day they were anaesthetized with Triethylamine (TEA) FlyNap ${ }^{\circledR}$ (Blades Biological Limited, Edenbridge, UK) and separated into groups of blood-fed and non-blood-fed specimens. For the processing of specimens and assessment of vector competence (infection, dissemination and transmission rates), a modified protocol adapted from $[15,19,28]$ was followed. Briefly, at 7 and $14 \mathrm{dpi}$, mosquitoes were immobilized at $-80^{\circ} \mathrm{C}$ for 2 mins and held in a plastic pot embedded in ice, to ensure that they remained immobile during processing. Legs and wings were removed, saliva samples were taken and the bodies, legs, wings and saliva retained for downstream analysis.

\section{Processing of samples for molecular detection of JEV RNA}

The protocol of [15] was used for detection of JEV RNA in tissues using previously published primers [29], which target and amplify a fragment of the NS1 gene. A sample was considered positive for JEV RNA at a Cycle threshold $(c t)$ value of 39 or lower.

\section{Immunohistochemistry}

The presence of JEV antigen in mosquitoes was determined by immunohistochemistry (IHC) in histological sections. Briefly, twelve infected blood-fed and three non-infected control female mosquitoes were placed in $10 \%$ neutral buffered formalin for fixation for $48 \mathrm{~h}$. After fixation the wings and extremities 
were removed and placed in sagittal plane prior to routine processing to paraffin blocks. Serial 3- $\mu$ m-thick sections of mosquitoes were cut and placed on silane-coated slides (3-trietoxysilyl-propylamine). Proteinase enzyme buffer (DAKO, Ely, Cambridgeshire, UK) applied for $15 \mathrm{~min}$ at $20^{\circ} \mathrm{C}$ was used as antigen retrieval method. A mouse monoclonal anti-Flavivirus E-glycoprotein antibody (ab155882, Abcam, Cambridge, UK) applied at 1 in 50 dilution in Tris Buffered saline with 0.05\% Tween 20 (TBST, VWR, Leicestershire, UK) at $4^{\circ} \mathrm{C}$ for $18-20 \mathrm{~h}$ (overnight) was used as primary antibody to detect JEV. Parallel sections were tested with a protein concentration matched mouse immunoglobulin $\mathrm{G}$ class $2 \mathrm{a}$ (Abcam, Cambridge, UK), as isotype controls in order to identify any non-specific immunolabelling. Slides were then washed in purified water and assembled into coverplates for immunolabelling. DAKO mouse EnVision $^{\text {TM }}+$ System, HRP Peroxidase (DAKO, Ely, Cambridgeshire, UK) was used as a secondary antibody and incubated for $30 \mathrm{~min}$ at $20^{\circ} \mathrm{C}$ combined with swine and goat immune serum (Vector Laboratories, Peterborough, UK). Antibody binding was visualized using the chromogen 3,3'-diaminobenzidine (DAB) + substrate-chromogen which results in a brown-colored precipitate at the antigen site after $10 \mathrm{~min}$ incubation. Finally, sections were counterstained with Mayer's haematoxylin (HE) and mounted in Distyrene Plasticiser Xylene (DPX) mounting medium (TCS Bioscience, Buckingham, UK) for light microscopy. Sections of West Nile virus-infected mouse brain and JEV-infected Vero cells were used as positive controls for flavivirus immunostaining.

\section{Virus titration}

Titrations of both stock virus and virus in the infected blood meal were performed by plaque assay as previously described $[19,28]$.

\section{Statistical analysis}

The graphical output was carried in the R programme (http://www.R-project.org). A ttest was performed comparing the $c t$ values for RT-PCR between each species.

\section{Results}

We assessed the potential to vector JEV in different mosquito populations, Ae. albopictus originating from Italy and Spain, and $C x$. quinquefasciatus from Tanzania, to which it was provided an infectious blood meal containing JEV. A total of 81 females from Ae. albopictus originating from Italy successfully fed (28.9\%), while 106 females of Ae. albopictus originating from Spain fed (52.7\%) (Table 1). Conversely, only 79 females of $C x$. quinquefasciatus fed (37.4\%) (Table 2). Mortality was observed between 1 and 2 $\mathrm{dpi}$, then the survival of mosquitoes was relatively stable until $6 \mathrm{dpi}$ before declining towards 13-14 dpi, which is typical for vector competence experiments (Additional file: Figure S1). 
Table 1

Infection, dissemination and transmission of Aedes albopictus (Italy and Spain) following consumption of a blood meal containing Japanese encephalitis virus genotype III. Groups of mosquitoes were maintained at $25^{\circ} \mathrm{C}$ for the indicated time periods

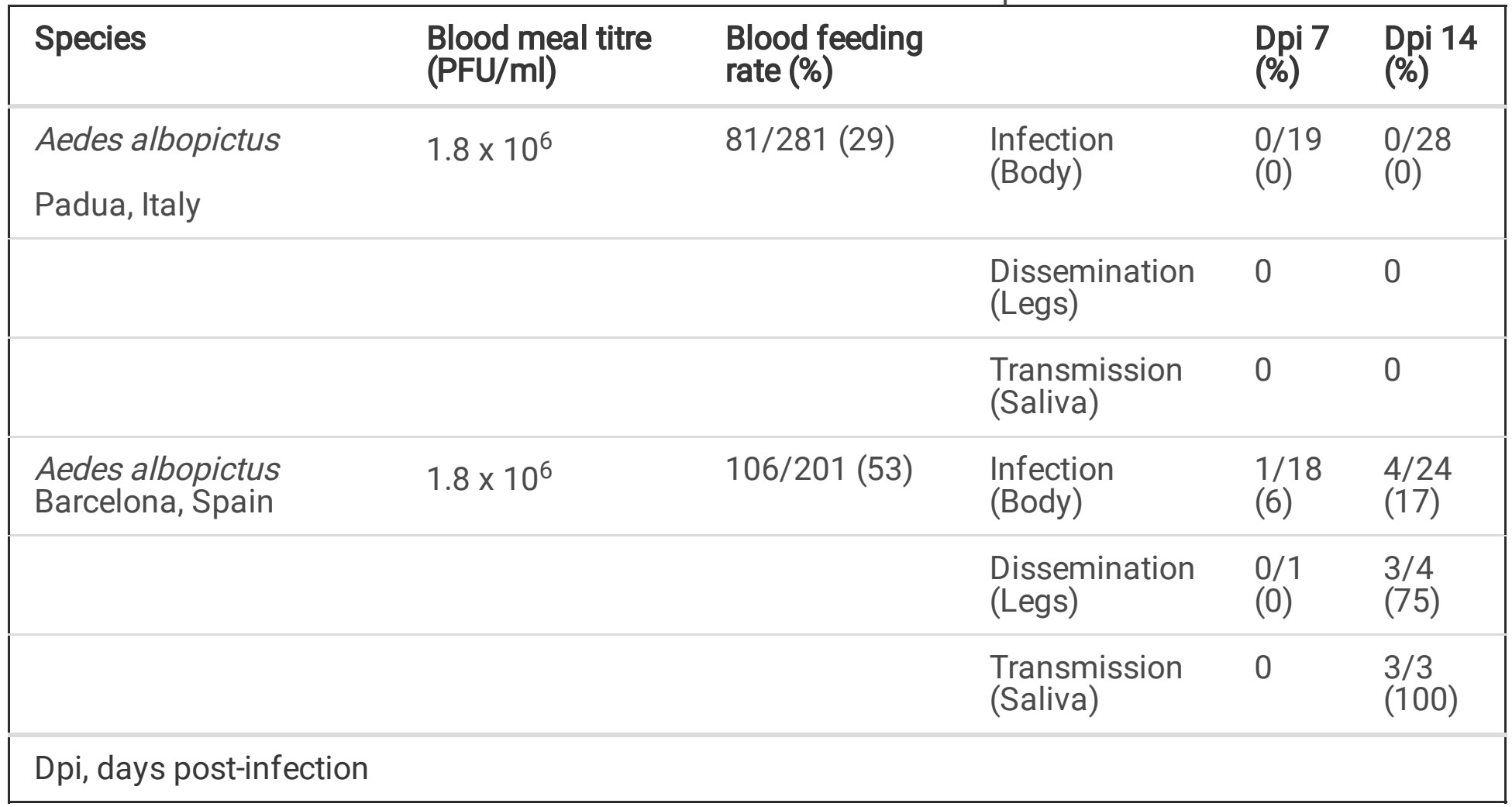

Table 2

Infection, dissemination and transmission of and Culex quinquefasciatus following consumption of a blood meal containing Japanese encephalitis virus genotype III. Groups of mosquitoes were maintained at $25^{\circ} \mathrm{C}$ for the indicated time periods

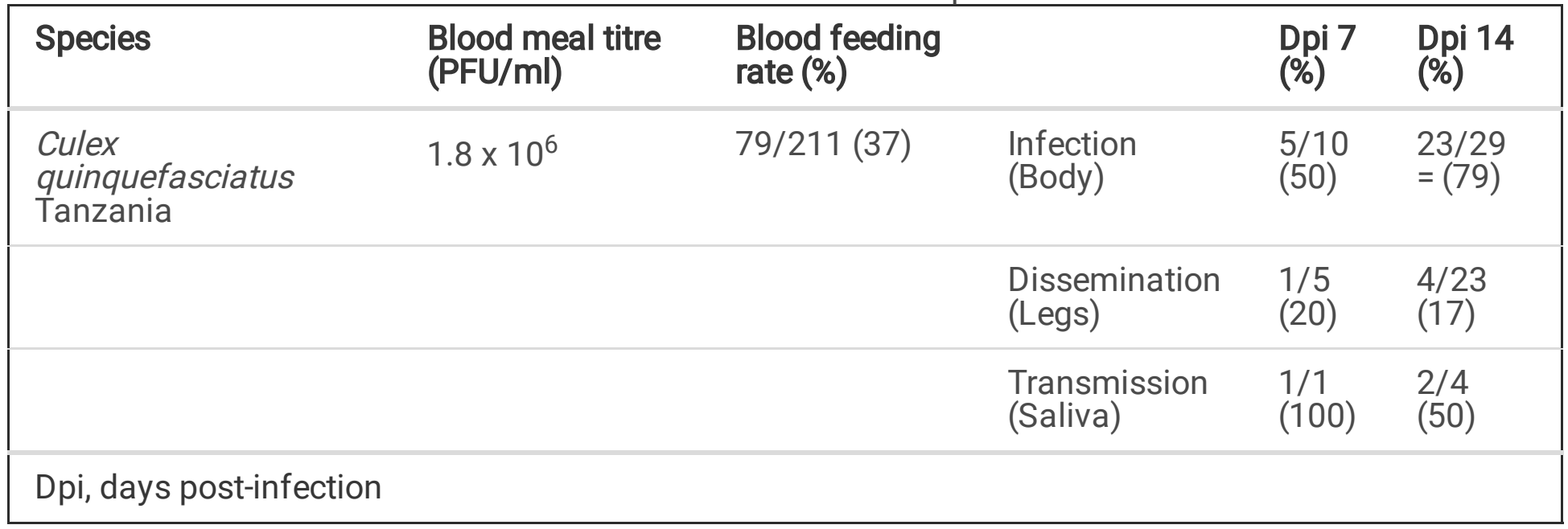

Virus infection, dissemination and transmission was initially determined by RT-PCR. Aedes albopictus originating from Italy did not show evidence for infection, dissemination or transmission at either 7 or 14 dpi (Table 1). However, Ae. albopictus originating from Spain did show infection at $7 \mathrm{dpi}$ (6\%), but neither dissemination nor transmission were detected. However, at 14 dpi infection (17\%), dissemination (75\%) and transmission $(100 \%, n=3)$ were detected (Table 1$)$. 
Culex quinquefasciatus showed higher prevalence of infection (50\%), dissemination (20\%) and transmission $(100 \%, n=1)$ at $7 \mathrm{dpi}$. In addition, higher prevalence of infection $(79 \%)$, dissemination (17\%) and transmission $(50 \%, n=2)$ were observed at $14 \mathrm{dpi}$ (Table 2). Of the mosquito populations assessed, $C x$. quinquefasciatus appeared the more competent vector for JEV, demonstrating the highest levels of dissemination and transmission, despite bloodfeeding at a lower rate than Ae. albopictus originating from Spain. However, these results demonstrated that a population of Ae. albopictus originating from Spain, may act as a competent vector for JEV.

To compare relative amounts of virus genome in the tissue we analysed, the threshold values from each amplification cycle (ct) were evaluated, in particular at $14 \mathrm{dpi}$ (Fig. 2). The $c t$ values detected from $C x$. quinquefasciatus were significantly lower compared to Ae. albopictus, suggesting a higher level of viral RNA detection in these samples $(t=2,163, p=0.019)$.

Cellular distribution of JEV infection in $C X$. quinquefasciatus was determined by IHC on sections of FFPE whole mosquitoes to detect JEV envelope antigen. All segments of the mosquito, head, thorax and abdomen, were examined by light microscopy. JEV-immunolabeled cells were observed in the posterior midgut of seven infected females of $C x$. quinquefasciatus (Fig. 3; Additional file, Figure S2). Immunolabelling was present in clusters of epithelial cells, predominantly ciliated pseudostratified intestinal cells, located in the posterior midgut region, as characterised by dark brown pigment deposition within the cytoplasm. The levels of midgut epithelial cells infection were highly variable with labelling ranging from a continuous row of cells to single cells (Additional file, Figure S2). Positive intracytoplasmatic immunolabelling was observed in the the salivary gland of one specimen of $C x$. quinquefasciatus, defined by the presence of secretory masses corroborating the detection of virus by this and other methods such as PCR (Table 2). Isotype control immunolabelled sections did not show any non-specific staining on infected mosquitoes.

\section{Discussion}

Our results show that $A e$. albopictus originating from Spain and $C x$. quinquefasciatus originating from Tanzania were susceptible to infection by JEV genotype III. In addition, our study demonstrates that after fourteen days at $25^{\circ} \mathrm{C}, \mathrm{JEV}$ virus was able to disseminate throughout Ae. albopictus originating from Spain and $C x$. quinquefasciatus from Tanzania with viral RNA being detected in their saliva by RT-PCR, and also in the salivary glands of $C x$. quinquefasciatus by IHC. This suggests that the studied populations may be competent vectors for JEV genotype III under our experimental conditions, corroborating previous findings from populations of Ae. albopictus and Cx. quinquefasciatus [13, 16, 21, $23,24,26,30]$.

Both Ae. albopictus and $C x$. quinquefasciatus are indigenous in tropical areas, where temperatures above $30^{\circ} \mathrm{C}$ can be encountered, and they are well known vectors for many arboviruses [24, 31-34]. Although 
$C x$. quinquefasciatus has not yet been detected in Europe, its morphological, ecological and phylogenetic similarity with $C x$. pipiens, and its ability to colonize new areas via ship and airline vessels [35], makes it a potentially important invasive species for studying the transmission of re-emerging zoonotic viruses such as JEV. The origin of $C x$. quinquefasciatus is thought to be West Africa from where it colonized other regions due to trade and migration. The species reached the Americas during the 1800 s, spreading to Asia and the Pacific via whaling and merchant vessels [36, 37]. It is a common species in Africa, thus our finding that the population from Tanzania is a highly competent vector for JEV is epidemiologically relevant in the event of JEV incurring in the African continent.

Conversely, Ae. albopictus is now widely distributed in many countries in continental Europe and the Mediterranean basin, with a few sporadic recorded incursions into more temperate regions such as the United Kingdom [38]. The species is widely distributed in Italy, Spain, and Germany, and several populations of this species are considered the main drivers for outbreaks of dengue and chikungunya in Europe [39]; it is also a known secondary vector of Zika virus in Latin America [28]. The species has spread rapidly throughout Europe, mainly due to being transported by road vehicles, where it is now a well-known biting pest together with Ae. japonicus japonicus [40]. In our study, the Italian population of Ae. albopictus from Padua, Northern Italy, was not a competent vector for JEV genotype III, although it is an efficient vector of other arboviruses such as chikungunya virus [41]. Previous reports have suggested that the different origins of the Italian Ae. albopictus populations which were introduced separately from different tropical and subtropical areas over the past three decades [41], could be the basis for differences in their vector competence. It is worth noting that the experimental conditions in this study maintained constant heat and humidity with a 24-hour day night photoperiod, which are standard conditions during vector competence studies $[15,19]$. However, these conditions are not representative of natural conditions. Given that this and other studies suggest that temperature appears to be critical for both vector competence and vector mortality in this experimental system, future studies would need to incorporate local variation between minimum and maximum temperature/humidity means, to relate it with what occurs in nature. Infection experiments carried out in $C X$. pipiens have shown that a limiting factor at which this species becomes unable to transmit JEV genotype III is temperature, with higher temperatures $\left(25^{\circ} \mathrm{C}\right)$ causing increased mortality in infected mosquitoes, when compared to mosquitoes held at $20^{\circ} \mathrm{C}$ [15]. However, there was no increased mortality observed for Ae. albopictus or $C x$. quinquefasciatus at $25^{\circ} \mathrm{C}$ in the present study, suggesting that under our experimental conditions an elevated temperature and infection with JEV strain SA-14 did not cause additional mosquito mortality. This may be a consequence of the mosquito species and virus strain used [3].

The labelling of virus antigen in $C x$. quinquefasciatus confirmed that at $25^{\circ} \mathrm{C}$, JEV was able to infect the posterior midgut epithelial cells such as ciliated pseudostratified intestinal cells, which corroborates detection of virus in the mosquito body by molecular means. This supports a previous study showing that midgut epithelial cells are a major site of viral replication [15]. In addition, viral antigen was observed in mid-gut and salivary glands by $\mathrm{IHC}$, which demonstrated that at $25^{\circ} \mathrm{C}$ and by $14 \mathrm{dpi}$, the virus was able to overcome the midgut barrier and to infect secondary organs such as the salivary glands. Previous 
studies found that JEV present in the midgut appeared viable by the recovery of live virus in vitro from homogenised mosquito bodies [15]. However, it was unclear whether the restriction of JEV to the midgut was a result of active anti-viral control by the mosquitoes or the lower experimental temperature restricting virus replication. The authors suggested that an increase in temperature, or an increase in the duration of the experiment, could potentially trigger further virus replication and escape from the midgut, our results suggest that temperature may be a contributing factor in full viral dissemination.

\section{Conclusions}

Of the mosquito populations studied, there was no evidence that the virus could infect or disseminate within the Ae. albopictus originating from Italy at $25^{\circ} \mathrm{C}$ at either 7 or $14 \mathrm{dpi}$. By contrast, Ae. albopictus originating from Spain and $C x$. quinquefasciatus originating from Tanzania proved to be susceptible to infection as early as $7 \mathrm{dpi}$. Dissemination occurred in a proportion of infected mosquitoes and JEV was detected in the saliva of these mosquitoes, suggesting the potential of these mosquito populations to transmit JEV genotype III (strain SA-14). Considering that several mosquito species have been shown to be competent vectors for a number of arboviruses, our results contribute to this expanding dataset and indicate that if JEV were to emerge in new areas, there are a number of mosquito populations that could facilitate its transmission and persistence.

\section{Declarations}

\section{Ethics approval and consent to participate}

Not applicable.

\section{Consent for publication}

Not applicable.

\section{Availability of data and materials}

All data generated by this study and used is presented within this published article.

\section{Competing interests}

The authors declare that they have no competing interests.

\section{Funding}

Funding was provided by the European Union Framework Horizon 2020 Innovation Grant European Virus Archive Global (EVAg, No. 653316) and the Department for Environment, Food and Rural Affairs (Defra), The Scottish Government and Welsh Government through grant SV3045 and SE4116.

\section{Author contributions}


NJ obtained funding for the study. LMHT, AJF, SS, FZXL, SA, AN, conceived and designed experiments. LMHT, AJF, SS, FZXL performed the experiments. LMHT, AJF, SS, FZXL, SA, AN, SB, AD, PV, KLM, and NJ analysed the data. LMHT wrote the first draft. NJ revised the draft and all authors contributed to and approved the final draft.

\section{Acknowledgements}

The authors thank Shahida Begum (London School of Hygiene and Tropical Medicine, United Kingdom) for the provision of $C x$. quinquefasciatus colony. Thanks are also giving to Jonas-Schmidt-Chanasit (Bernhard Nocht Institute, Hamburg, Germany) for providing the JEV (strain SA-14).

\section{Authors details}

${ }^{1}$ Vector Borne Diseases Research Group, Animal and Plant Health Agency, Addlestone, Surrey, UK. ${ }^{2}$ Pathology Department, Animal and Plant Health Agency, Addlestone, Surrey, United Kingdom. ${ }^{3}$ Veterinary Faculty, University of Zaragoza, Zaragoza, Spain. ${ }^{4}$ Entostudio, Viale del Lavoro, Ponte San Niccolo, Padova, Italy. ${ }^{5}$ Rabies and Viral Zoonosis Research Group, Animal and Plant Health Agency, Addlestone, Surrey, UK. ${ }^{6}$ Faculty of Health and Medicine, University of Surrey, Surrey, United Kingdom

\section{References}

1. Alkan C, Zapata S, Bichaud L, Moureau G, Lemey P, Firth AE, et al. Ecuador Paraiso Escondido virus, a new flavivirus isolated from New World sand flies in Ecuador, is the first representative of a novel clade in the genus Flavivirus. J Virol. 2015;89:11773-85; doi:10.1128 /JVI.01543-15.

2. Braack L, Gouveia de Almeida AP, Cornel AJ, Swanepoel R, Jager C. Mosquito-borne arboviruses of African origin: review of key viruses and vectors. Parasit Vectors. 2018;11:29. doi.org/10.1186/s13071-017-2559-9.

3. Auerswald H, Maquart P-O, Chevalier V, Boyer S. Mosquito vector competence for Japanese Encephalitis virus. Viruses. 2021;13:1154. doi.org/10.3390/v13061154.

4. Schuh AJ, Ward MJ, Brown AJL, Barrett ADT. Phylogeography of Japanese encephalitis virus: Genotype is associated with climate. PLoS Negl Trop Dis. 2014;7:e2411.

5. Mansfield KL, Hernández-Triana LM, Banyard AC, Fooks AR, Johnson N. Japanese encephalitis virus infection, diagnosis and control in domestic animals. Veter Microb. 2017;201:85-92.

6. Hameed M, Wahaab A, Nawaz M, Khan S, Nazir J, Liu K, et al. Potential role of birds in Japanese encephalitis virus zoonotic transmission and genotype shit. Viruses. 2021;13:357; doi.org /10.3390/v13030357.

7. Ricklin ME, García-Nicolás O, Brechbühl D, Python S, Zumkehr B, Nougairede A, et al. Vector free transmission and persistence of Japanese encephalitis virus in pigs. Nat Comm. 2016;7:10832.

8. Gould EA, Higgs S, Buckley A, Gritsun TA. Potential arbovirus emergence and implications for the United Kingdom. Emerg Infect Dis. 2006;12:549-55. 
9. Pearce JC, Learoyd TP, Langendorf BJ, Logan JG. Japanese encephalitis: The vectors, ecology and potential for expansion. J Travel Med. 2018;25:16-26.

10. Oliveira ARS, Strathe E, Etcheeverry L, Cohnstaedt LW, McVey DS, Piaggio J, et al. Assessment of data on vector and host competence for Japanese encephalitis virus: A systemic review. Prevent Vet Med. 2018;54:71-89.

11. Lytra I, Emmanouel N. Study of Culex tritaeniorhynchus and species composition of mosquitoes in a rice field in Greece. Acta Trop. 2014;134:66-71.

12. Ravanini P, Huhtamo E, llaria V, Nicosia AM, Servivo L, Rivasi F, et al. Japanese encephalitis virus RNA detected in Culex pipiens mosquitoes in Italy. Eurosurveill. 2012;17:pii = 20221. doi.org/10.2807/ese.17.20221-en.

13. Mackenzie-Imponvil L, Impoinvil DE, Galbraith SE, Dillon RJ, Ranson H, Johnson N, et al. Evaluation of a temperate climate mosquito, Ochleratutus detritus (= Aedes detritus), as a potential vector of Japanese encephalitis virus. Med Vet Entomol. 2015;29:1-9. doi:10.1111/mve.12083.

14. Chapman GE, Sherlock K, Hesson JC, Blagrove MS, Lycett GJ, Debra Archer D, et al. Laboratory transmission potential of British mosquitoes for equine arboviruses. Parasit Vectors. 2021;13:413. doi.org/10.1186/s13071-020-04285-x.

15. Folly AJ, Dorey-Robinson D, Hernández-Triana LM, Ackroyd S, Vidana B, Lean FZXL, et al. Temperate conditions restrict Japanese encephalitis virus infection to the mid-gut and prevents systemic dissemination in Culex pipiens mosquitoes. Scient Rep. 2021;11:6133. doi.org/10.1038/s41598-021-85411-2.

16. de Wispelaere M, Desprès P, Choumet V. European Aedes albopictus and Culex pipiens are competent vectors for Japanese encephalitis virus. PLoS Negl Trop Dis. 2017;11:e0005294. doi:10.1371/journal.pntd.0005294.

17. Huber K, Jansen S, Leggewie M, Badusche M, Schmidt-Chanasit J, Becker N, et al. Aedes japonicus japonicus (Diptera: Culicidae) from Germany have vector competence for Japan encephalitis virus but are refractory to infection with West Nile virus. Parasitol Res. 2014;113:3195-9; doi.10.1007/s00436-014-3983-9.

18. Gunay F, Alten B, Simsek F, Aldemir A, Linton T-M. Barcoding Turkish Culex mosquitoes to facilitate arbovirus vector incrimination studies reveals hidden diversity and new potential vectors. Acta Trop. 2015;43:112-20.

19. Hernández-Triana LM, Barrero E, Delacour-Estrella S, Ruiz-Arrondo I, Lucientes J, Fernández de Marco MMF, et al. Evidence for infection but not transmission of Zika virus by Aedes albopictus (Diptera: Culicidae) from Spain. Parasit Vectors. 2019;12:204; doi.org/10.1186/ s13071-019-3467-y.

20. Schaffner F, Medlock JM, Van Bortel W. Public health significance of invasive mosquitoes in Europe. Clin Microbiol Infect. 2013;19:685-92.

21. Nicholson J, Ritchie SA, van den Hurk AF. Aedes Albopictus (Diptera: Culicidae) as a potential vector of endemic and exotic arboviruses in Australia. J Med Entomol. 2014;51:661-9. 
22. Chen W-J, Dong C-F, Chiou L-Y, Wen-Ling Chuang W-L. Potential role of Armigeres subalbatus (Diptera: Culicidae) in the transmission of Japanese encephalitis virus in the absence of rice culture on Liu-Chiu Islet, Taiwan. J Med Entomol. 2000;37:108-13;. doi.org/10.1603/0022-2585-37.1.108.

23. Weng MH, Lien JC, Wang YM, Wu HL, Chin C. Susceptibility of three laboratory strains of Aedes albopictus (Diptera: Culicidae) to Japanese encephalitis virus from Taiwan. J Med Entomol. 1997;34:745-7.

24. Huang YJ, Harbin JN, Hettenbach SM, Maki E, Cohnstaedt LW, Barret ADT, et al. Susceptibility of a North American Culex quinquefasciatus to Japanese encephalitis virus. Vector-Borne Zoon Dis. 2015;15:709-11. doi:10.1089/vbz.2015.1821.

25. Banerjee K, Deshmukh PK, Ilkal MA, Dhanda V. Comparative susceptibility of three species of mosquitoes to infection with Japanese encephalitis virus. Indian J Med Res. 1983;78:603-6.

26. van den Hurk AF, Nisbet DJ, Hall RA, Kay BH, Mackenzie JS, Ritchie SA. Vector competence of Australian mosquitoes (Diptera: Culicidae) for Japanese encephalitis virus. J Med Entomol. 2003;40:82-90.

27. Kramer LD, Chin P, Cane RP, Kauffman EB, Mackereth G. Vector competence of New Zealand mosquitoes for selected arboviruses. Am J Trop Med Hyg. 2011;85:182-9.

28. Hernández-Triana LM, Fernández de Marco M, Mansfield KL, Thorne L, Lumley S, Marston D, et al. Assessment of vector competence of UK mosquitoes for Usutu virus of African origin. Parasit Vectors. 2018;11:381; doi.org/10.1186/s13071-018-2959-5.

29. Pyke TA, Smith IL, van den Hurk AF, Northill JA, Chuan TF, Westacott A, et al. Detection of Australasian Flavivirus encephalitic viruses using rapid fluorogenic TaqMan RT-PCR assays. J Virol Methods. 2014;117:161-7.

30. Hanna JN, Ritchie SA, Phillips DA, Lee JM, Hills SL, van den Hurk AF, et al. Japanese encephalitis in North Queensland, Australia, 1998. Med J Aust. 1999;170:533-6.

31. Bhattacharya S, Basu P. The southern house mosquito, Culex quinquefasciatus: profile of a smart vector. J Entomol Zool Studies. 2016;4:73-81.

32. Farajollahi A, Fonseca DM, Kramer LD, Kilpatrick AM. "Bird biting" mosquitoes and human disease: A review of the role of Culex pipiens complex mosquitoes in epidemiology. Infect Gen Evol. 2011;7:1577-85. doi.org/10.1016/j.meegid.2011.08.013.

33. Mitchell CJ. The role of Aedes albopictus as an arbovirus vector. Parassitol. 2020;37:108-13.

34. Vega-Rúa A, Marconcini M, Madec Y, Manni M, Carraretto D, Gomulski LM, et al. Vector competence of Aedes albopictus populations for chikungunya virus is shaped by their demographic history. Commun Biol. 2020. 3:326; doi.org/10.1038/s42003-020-1046-6.

35. Bataille A, Cunningham AA, Cedeño V, Cruz M, Eastwood G, Fonseca DM, et al Evidence for regular ongoing introductions of mosquito disease vectors into the Galápagos Islands. Proc Royal Soc London, Series B, Biol Scienc. 2009;276:3769-3775.

36. Belkin JN, Heinemann SJ. Collection records of the project "Mosquitoes of Middle America". 3. Bahama Is. (BAH), Cayman Is. (CAY), Cuba (CUB), Haiti (HAC, HAR, HAT) and Lesser Antilles (LAR). 
Mosq Syst. 1975;7:367-93.

37. Vinogradova EB. Culex pipiens pipiens mosquitoes, taxonomy, distribution, ecology physiology genetics, applied importance and control. Sofia: Pensoft; 2000. 646 pp.

38. Medlock JM, Vaux AGC, Cull B, Schaffner F, Gillingham E, Pfluger V, et al. Detection of the invasive mosquito species Aedes albopictus in southern England. The Lancet. 17:140.

39. Jourdain F, Roiz D, de Valk H, Noël H, L'Ambert G, Franke F, et al. From importation to autochthonous transmission: Drivers of chikungunya and dengue emergence in a temperate area. PLoS Negl Trop Dis. 2020;14:e0008320. doi.org/10.1371/journal.pntd.0008320.

40. Werner D, Kampen H. The further spread of Aedes japonicus japonicus (Diptera, Culicidae) towards northern Germany. Parasitol Res. 2013;112:3665-8. doi.org/10.1007/s00436-013-3564-3.

41. Severini F, Boccolini D, Fortuna C, Di Luca M, Toma L, Amendola A, et al. Vector competence of Italian Aedes albopictus populations for the chikungunya virus (E1-226V). PLoS Negl Trop Dis. 2018;12:e0006435. doi.org/10.1371/journal.pntd.0006435.

42. Perry A, Golding N. 2021. Range of environmental temperature conditions in the United Kingdom. Met Office Report for Transport for London, 63 pp. [last accessed 10.Dec.2021].

\section{Figures}




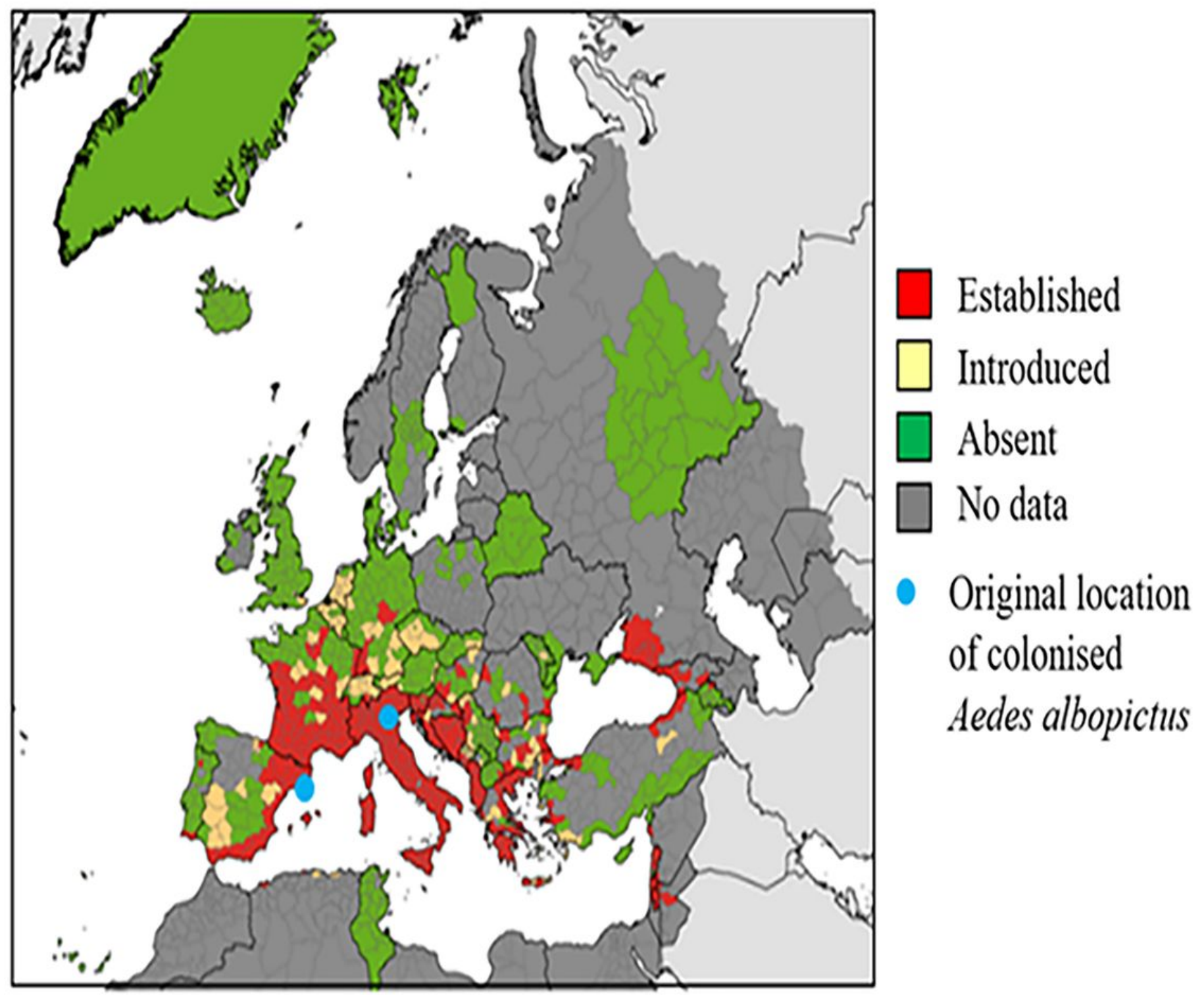

Figure 1

Map of Europe showing distribution Aedes albopictus and indicating the sites where the colonised strains were originally trapped (blue circle). Map published by European Centre for Disease Prevention (modified from https://www.ecdc.europa.eu/en/publications-data/aedes-albopictus-current-known-distributionmarch-2021). 


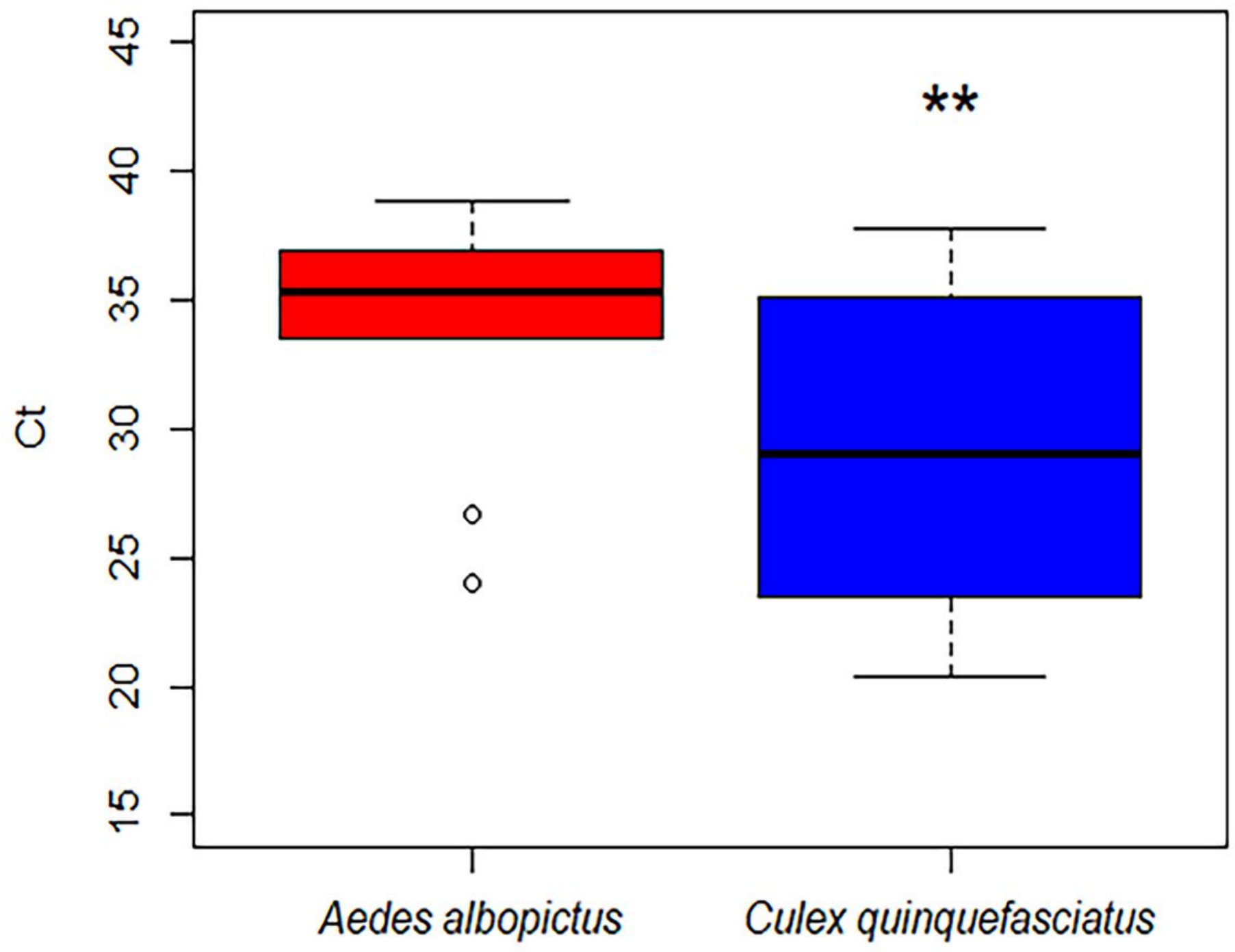

Species

Figure 2

Boxplot comparing the cycle threshold ( $c t$ ) values for Aedes albopictus and Culex quinquefasciatus from RNA extractions of specimens infected with Japanese encephalitis virus and maintained at $25^{\circ} \mathrm{C}$. Culex quinquefasciatus ct values were significantly lower compared to Ae. albopictus, suggesting that quantity of viral RNA was higher in these samples. Significant difference denoted by a double asterisk ( $\left.{ }^{\star *}\right)$. 

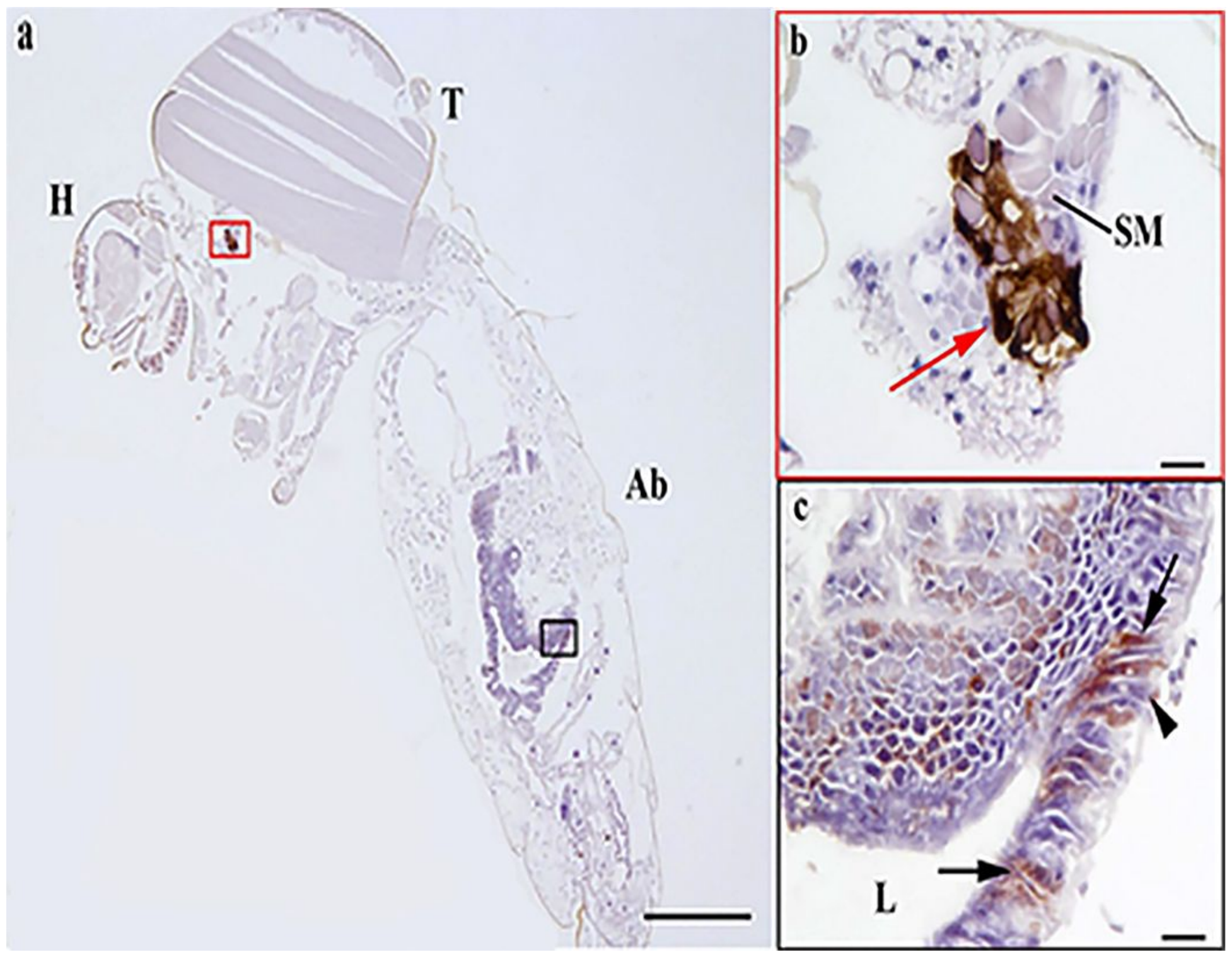

\section{Figure 3}

Japanese encephalitis virus infection at $25^{\circ} \mathrm{C}$ of posterior midgut epithelial cells in $\mathrm{Cx}$. quinquefasciatus. (a) Head $(H)$, thorax $(T)$, abdomen (Ab). (b) Intracytoplasmic immunolabelling in the distal lobes of salivary gland, defined by the presence of secretory masses (SM); intense antigen labelling particularly in the basal region of the epithelium (red arrow). (c) Antigen labelling in the apical ciliated cells (arrow) and basal epithelial cells (arrowhead) of the posterior midgut; lumen of the midgut (L). Scale bar: $500 \mu m(a)$; $20 \mu \mathrm{m}(\mathrm{b}, \mathrm{c})$.

\section{Supplementary Files}

This is a list of supplementary files associated with this preprint. Click to download.

- GraphicalabstractmodifiedJEVInvasivesppHernandezTr.jpg 
- AdditionalFileS1HernandezTretal.jpg

- AdditionalFileS2HernandezTretal.jpg 